\title{
Androgen receptor functions as a negative transcriptional regulator of DEPTOR, mTOR inhibitor
}

\author{
Yuichiro Kanno', Shuai Zhao', Naoya Yamashita', Kazuyuki Yanai², Kiyomitsu Nemoto' \\ and Yoshio Inouye ${ }^{1}$ \\ ${ }^{1}$ Department of Molecular Toxicology, Faculty of Pharmaceutical Sciences, Toho University, \\ Funabashi, Chiba 274-8510, Japan \\ ${ }^{2}$ Department of Biomolecular Science, Faculty of Science, Toho University, Funabashi, Chiba 274-8510, Japan
}

(Received July 21, 2015; Accepted September 5, 2015)

\begin{abstract}
It has been noticed that crosstalk between androgen receptor (AR) and mammalian target of rapamycin (mTOR) signaling pathways plays a crucial role in the proliferation of prostate cancer cells. To clarify this mechanism, we focused on DEPTOR, a naturally occurring inhibitor of mTOR. The treatment of a human AR-positive prostate cancer cell line, LNCaP, with the AR-agonist dihydrotestosterone (DHT) repressed DEPTOR mRNA expression in a time-dependent manner. This repression was abrogated by treatment with the AR-antagonist bicalutamide. Knockdown of DEPTOR mRNA by siRNA resulted in the increased phosphorylation of $70 \mathrm{kDa}$ ribosomal protein S6 kinase 1 (S6K), a substrate of mTORC1, accompanied by the elevated expression of cyclin D1, a positive regulator of cell proliferation. Furthermore, the ChIP assay demonstrated that AR could bind to AR-responsible element-like region within the 4th intron of the DEPTOR gene. The amount of acetylated histone H3 (Lys9, Lys14) was reduced by the DHT treatment in this region. Taken together, these results propose that AR-dependent prostate cancer cell proliferation requires decreased DEPTOR transcription directly controlled by AR.
\end{abstract}

Key words: Androgen receptor, mTOR, DEPTOR, Prostate cancer

\section{INTRODUCTION}

Androgen receptor (AR) is a member of the nuclear receptor (NR) superfamily of ligand-regulated transactivation factors. AR mediates various biological effects such as the development of male reproductive tissues, sexual development, and spermatogenesis (Matsumoto et al., 2003, 2005, 2008; Lee and Chang, 2003). AR also plays a central role in prostate cancer growth and development (Heinlein and Chang, 2004; Dehm and Tindall, 2007; Bennett et al., 2010). In the absence of ligand, AR is localized in the cytoplasm, where it forms a complex with chaperones. Upon binding of ligands, such as testosterone and 5- $\alpha$-dihydrotestosterone (DHT), AR translocates into the nucleus. Following nuclear translocation, AR homodimer binds to androgen responsive elements (AREs) in the promoter regions of its target genes.

The mammalian target of rapamycin (mTOR) is an atypical serine/threonine protein kinase, interacting with several proteins to form two distinct complexes (mTORC1 and mTORC2) (Laplante and Sabatini, 2012).
mTORC1 mainly regulates mRNA translation, cell size and cell proliferation by phosphorylation of $70 \mathrm{kDa}$ ribosomal protein S6 kinase 1 (S6K) and eukaryotic translation initiation factor 4E (eIF4E), while mTORC2 regulates cell survival by activation of AKT and the serum and glucocorticoid inducible kinase 1 (SGK1) (Guertin and Sabatini, 2007).

Recently, it was reported that the crosstalk between AR and mTOR played an important role in prostate cancer cell proliferation (Xu et al., 2006). DHT activated mTORC1 and subsequently increased translation of cell cycle positive regulator, cyclin $\mathrm{D} 1$, in $\mathrm{LNCaP}$ prostate cancer cells. However, the detailed mechanism of AR-dependent regulatory mechanism of $\mathrm{mTOR}$ is not yet clear. DEP domain-containing mTOR-interacting protein (DEPTOR) was identified as a naturally occurring inhibitor of mTOR in both mTORC1 and mTORC2 (van Duin et al., 2005; Peterson et al., 2009). DEPTOR is suggested to be a tumor suppressor due to the inhibitory effect on mTORC1 and mTORC2 pathways. Recently, the post-translational regulation of DEPTOR was reported

Correspondence: Yuichiro Kanno (E-mail: ykanno@phar.toho-u.ac.jp) 
(Peterson et al., 2009; Li et al., 2014; Zhao et al., 2011; Gao et al., 2011). DEPTOR was phosphorylated by casein kinase 1 alpha, facilitating ubiquitylation by the $\mathrm{F}$ box protein SCF ( $\beta$ TrCP) (Duan et al., 2011). Although post-translational regulation of DEPTOR expression has been investigated, transcriptional regulation of DEPTOR remains unclear.

In this study, we examined whether the DEPTOR gene was regulated by AR. We show that DHT down-regulates the transcription of the DEPTOR gene by promoting the binding of AR to intronic ARE in prostate cancer cells. This AR-mediated transrepression of the DEPTOR gene is a novel finding to explain the mechanism of the crosstalk between AR and mTOR signaling pathways.

\section{MATERIAL AND METHODS}

\section{Chemicals}

Dihydrotestosterone (DHT), bicalutamide and cycloheximide (CHX) were purchased from Sigma Aldrich (St. Louis, MO, USA).

\section{Cell culture}

Androgen-receptor-negative and -positive prostate cancer cell lines, PC-3 and LNCaP, respectively, and bone-derived cell line, vertebral-cancer of the prostate (VCaP), were cultured in RPMI 1640 medium (Wako, Osaka, Japan) supplemented with 10\% fetal bovine serum (FBS) and penicillin-streptomycin in a humidified atmosphere containing $5 \% \mathrm{CO}_{2}$ at $37^{\circ} \mathrm{C}$. The cells were treated as described in the figure legends.

\section{Western blotting}

Total protein was extracted with SDS sample buffer supplemented with protease inhibitor cocktail and the protein concentration was measured with the Pierce 660 nm Protein Assay Reagent (Thermo Scientific, Hudson, NH, USA). The protein samples were separated by SDS-PAGE. Western blotting was performed with rabbit anti-DEPTOR, anti-pS6K, anti-S6K and anti-cyclin D1 antibodies (Cell Signaling Technology, New York, NY, USA) as primary antibodies and horseradish peroxidaseconjugated anti-rabbit IgG (Cell Signaling) as a secondary antibody. Anti- $\alpha$-tubulin pAb-HRP-DirecT (MBL, Aichi, Japan) antibody was used as loading control. Protein bands were visualized using the Immobilon Western Detection Reagent (Millipore, Billerica, MA, USA) and then the signals were captured by Typhoon 9500 (GE Healthcare, Piscataway, NJ, USA). Band density was measured by ImageJ software.

\section{Realtime RT-PCR}

The cells were seeded in 12-well plates in phenol redfree RPMI containing 5\% charcoal-stripped FBS (csFBS; GIBCO, Grand Island, NY, USA). On the next day, the cells were treated with DHT for $24 \mathrm{hr}$. Total RNA was isolated using ISOGEN II (Nippon Gene, Tokyo, Japan). cDNA was synthesized using a ReverTra Ace qPCR RT Kit (Toyobo, Osaka, Japan). Quantitative RTPCR (qRT-PCR) was conducted using KOD SYBR qPCR Mix (Toyobo) according to the manufacturer's protocol and using the 7500 Fast Real-Time PCR System Software (Applied Biosystems, Foster City, CA, USA). The following pairs of primers were used for FKBP51(forward 5'- TCCCTCGAATGCAACTCTCT -3', reverse 5'- GCCACATCTCTGCAGTCAAA -3'), DEPTOR (forward 5'- AAGCTGATGAGCCCTGAAAA -3', reverse 5'- TTGTTGGACACATGCTGGAT -3') and $\beta$ -actin (forward 5'-TCCTCCTGAGCGCAAGTACTC-3', reverse 5'-CTGCTTGCTGATCCACATCTG-3').

\section{siRNA-induced mRNA knockdown}

LNCaP cells were transfected with DEPTOR-targeting or control small interfering RNAs (siRNAs; SigmaAldrich) using Lipofectamine RNAiMAX reagent (Invitrogen, Carlsbad, CA, USA) according to the manufacturer's instructions.

\section{Chromatin immunoprecipitation assay}

The LNCaP cells were seeded in $100 \mathrm{~mm}$ dishes in phenol red-free RPMI containing 5\% csFBS. On the next day, cells were treated with DHT or solvent for $6 \mathrm{hr}$. Chromatin immunoprecipitation (ChIP) assay was carried out using SimpleChIP Enzymatic Chromatin IP Kit (Cell Signaling Technology) according to manufacturer's protocol. Cells were fixed with $1 \%$ formaldehyde (Sigma Aldrich) for $15 \mathrm{~min}$ at $37^{\circ} \mathrm{C}$ and nuclei were prepared. Chromatins were digested with micrococcal nuclease to achieve a DNA ladder of 300-1,000 bp. The chromatins were immunoprecipitated with antiAR (Abcam, Cambridge, MA, USA), anti-Histone H3, and anti-Acetyl-Histone H3 (Lys9/Lys14) (Cell Signaling Technology) antibodies overnight at $4{ }^{\circ} \mathrm{C}$. The precipitated DNA was used for PCR amplification with the following specific primer pairs to ARE regions: FKBP5 ARE (5'-GGAGCCTCTTTCTCAGTTTTG-3' and 5'-CAATCGGAGTGTAACCACATC-3'), DEPTOR promoter (5' -CCATAGAAAAAGATACAGGC-3' and 5'-TCATTCCATGAAATTTGAATG-3'), DEPTOR ARE1 (5' '-TCCCCTCTTAATCTGGTTA-3' and 5' -CTAATGGCATACGGGCTTGT-3') and DEPTOR ARE2 (5'-TGAAGACGTTGAGGCATTTG-3' and 5'-ACAG- 
GGCCAAAGCATATGAG-3'). Band density was measured by ImageJ software.

\section{Statistical analysis}

Statistical comparisons were performed with one-way analysis of variance (ANOVA) followed by Dunnett's multiple comparison test as the post hoc test, and differences were considered statistically significant.

\section{RESULTS AND DISCUSSION}

\section{Androgen receptor dependent repression of DEPTOR mRNA expression in prostate cancer cell lines}

Crosstalk between AR and mTOR signaling pathways has been expected. DEPTOR is known as a natu- rally occurring inhibitor of mTOR via direct binding to both mTORC1 and mTORC2 (Peterson et al., 2009). Therefore, we investigated whether the expression of DEPTOR mRNA was regulated by AR in prostate cancer cell lines. LNCaP cells were treated with DHT for 3, 6 and $24 \mathrm{hr}$. The amount of DEPTOR mRNA was reduced by the DHT treatment in a time-dependent manner (Fig. 1A), whereas time-dependent transactivation of FKBP5 gene, one of AR-target genes, was observed. The DHT-dependent repression in DEPTOR mRNA was attenuated by co-treatment with the AR antagonist bicalutamide (Fig. 1B). Similar results were obtained in AR-positive prostate cancer VCaP cells (Fig. 1C). On the other hand, DHT-dependent alternation of DEPTOR mRNA was not observed in AR-negative prostate cancer PC-3 cells (Fig. 1D). The results suggest that negative regulation

A

LNCap

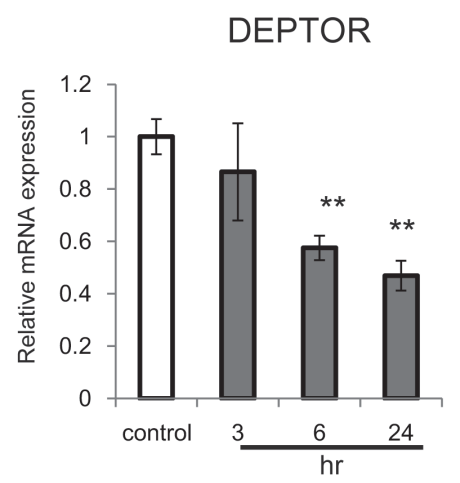

C

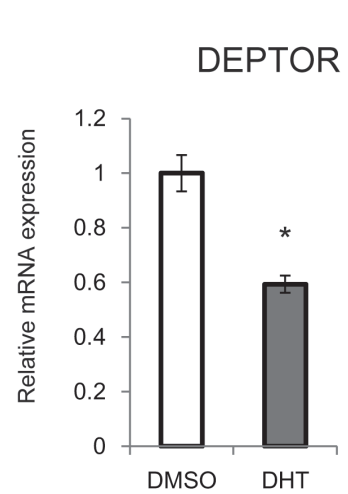

VCap

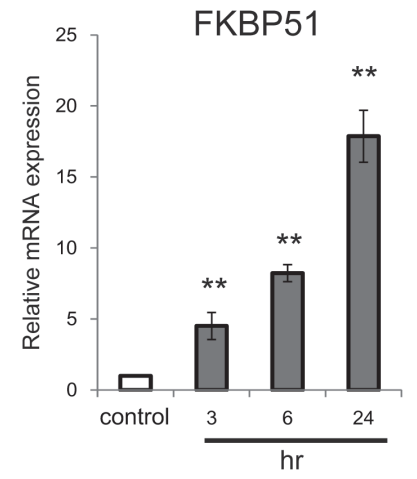

B

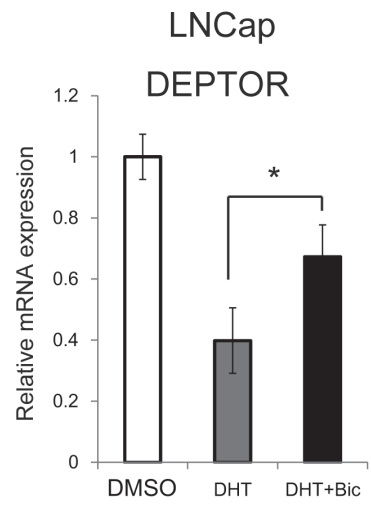

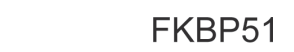

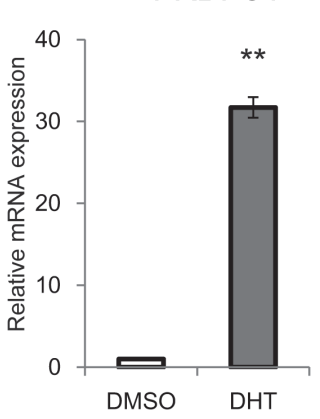

D

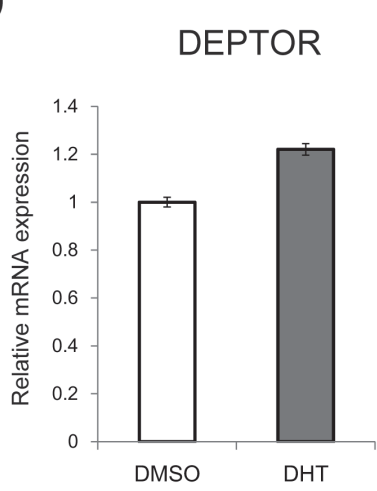

PC-3
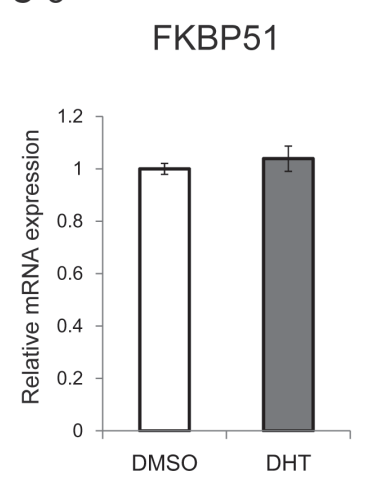

Fig. 1. Suppression of DEPTOR gene transcription by DHT in an AR-dependent manner. (A) LNCaP cells were treated with $10 \mathrm{nM}$ DHT for the indicated times. (B) LNCaP cells were treated with $10 \mathrm{nM}$ DHT with or without $10 \mu \mathrm{M}$ bicalutamide. (C, D) VCaP and PC-3 cells were individually treated with $10 \mathrm{nM}$ DHT for $24 \mathrm{hr}$. Cells were harvested and the mRNA levels of DEPTOR were measured by qRT-PCR, simultaneously with those of known AR-target gene FKBP51. Results normalized against those of $\beta$-actin are expressed as averaged fold activation over the solvent control of the four individual experiments (mean \pm S.D., $n=4$ ); $* P<0.05,{ }^{*} P P<0.01$. 
of DEPTOR mRNA expression by DHT requires AR in prostate cancer cells.

\section{Effect of DEPTOR mRNA knockdown on mTOR signaling pathway in prostate cancer cells}

DEPTOR protein expression was suppressed by the DHT treatment for $24 \mathrm{hr}$ in LNCaP cells (Fig. 2A). To confirm that $\mathrm{S} 6 \mathrm{~K}$ was activated by downregulation of DEPTOR, siRNA for DEPTOR was transfected in LNCaP cells. The siRNA-induced knockdown of DEPTOR mRNA raised not only the phosphorylation level of S6K but also the level of cyclin D1 protein, a positive regulator of cell proliferation (Fig. 2B). Various functions of DEPTOR in cancer progression have been reported. Ectopic expression of the DEPTOR gene is known to reduce $\mathrm{mTORC} 1$ activity and cell growth in pancreatic cancer cells (Li et al., 2014). Here, we showed that knockdown of DEPTOR mRNA enhanced phosphorylation degree of S6K and protein level of cyclin D1 in prostate cancer LNCaP cells. These observations suggest that downregulation of DEPTOR increases cell proliferation by DHT in AR-positive prostate cancer cells.

\section{Binding of AR to intronic ARE of DEPTOR gene}

Next, we investigated whether the repression of DEPTOR mRNA by DHT was regulated by de novo protein synthesis. The repressed expression of DEPTOR mRNA by DHT was observed even in the presence of cycloheximide (CHX) (Fig. 3A). This result suggests that the transrepression of the DEPTOR gene by DHT does not require the novel protein synthesis. By in silico, using published dataset of ChIP-on-chip analysis in LNCaP cells (Wang et al., 2009), we identified two putative AR binding regions, which were named ARE1 and ARE2, within the 3rd and 4th introns, respectively, of the DEPTOR gene (Fig. 3B). In order to estimate the binding potential of AR to ARE1 and ARE2, ChIP assay was carried out in the absence or presence of DHT in LNCaP cells (Fig. 3C). It was demonstrated that ARE2 was immunoprecipitated by antiAR antibody, but not the region or ARE1. Furthermore, the extent of acetylated histone H3 (Lys9, Lys14), which enhanced gene transactivation, was examined by ChIP assay. Whereas total histone H3 level remained unchanged, acetylated histone $\mathrm{H} 3$ level was reduced by the DHT treatment in ARE2 region (Fig. 3D). In contrast, acetylated histone $\mathrm{H} 3$ level at ARE of FKBP5 gene was enhanced. It is known that the histone acetylation leads to transcriptional activation. Especially, histone acetylation is dynamically controlled by histone acetyltransferases (HATs) and histone deacetylases (HDACs). Various negatively AR-regulated genes have been reported. In the case of E-cadherin (Liu et al., 2008), activated AR down-
A

B

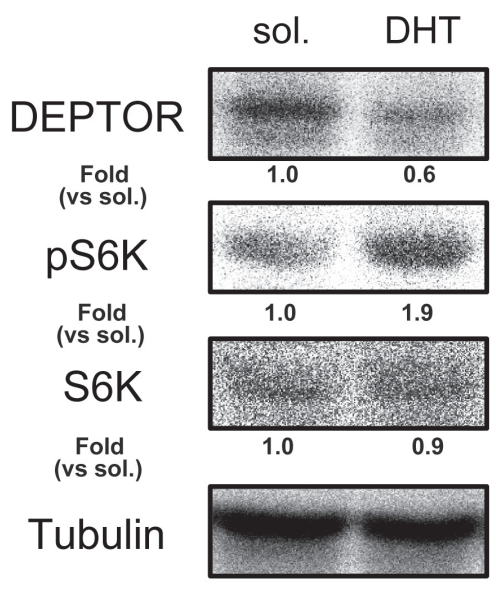

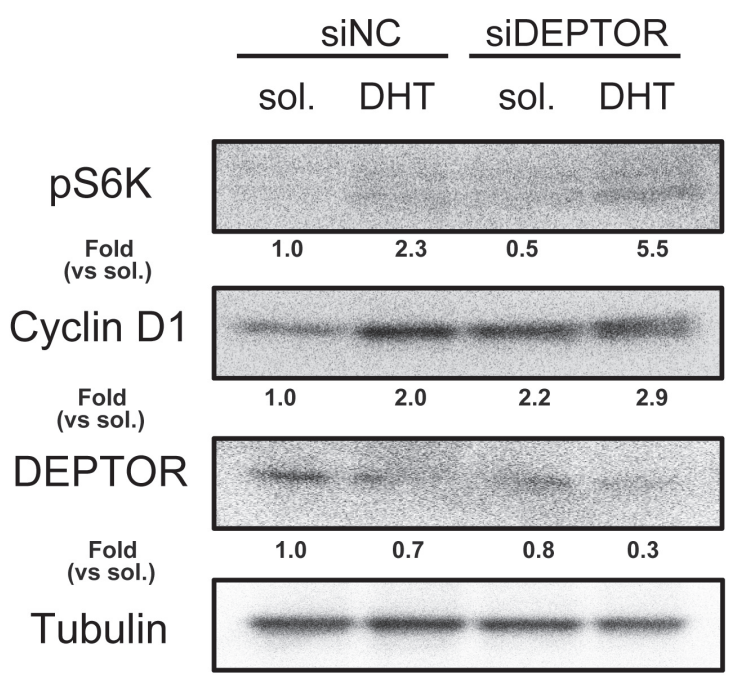

Fig. 2. Reduction in DEPTOR protein level by the DHT treatment and elevation in cyclin D1 protein level by knockdown of $D E P$ TOR gene. (A) LNCaP cells were treated with solvent control (sol.) or $10 \mathrm{nM}$ DHT for $24 \mathrm{hr}$. Whole-cell lysates were separated by SDS-PAGE, and proteins were detected by immunoblotting using antibodies against DEPTOR, pS6K(T389), S6K and tubulin used as a loading control. (B) LNCaP cells were transfected with DEPTOR-targeting or control siRNA. After $48 \mathrm{hr}$, cells were treated with DHT or sol. for an additional $24 \mathrm{hr}$. Whole-cell lysates were separated by SDS-PAGE, and proteins were detected by immunoblotting using antibodies against Cyclin D1, DEPTOR, pS6K(T389) and tubulin used as a loading control. 
AR downregulates DEPTOR mRNA expression

A

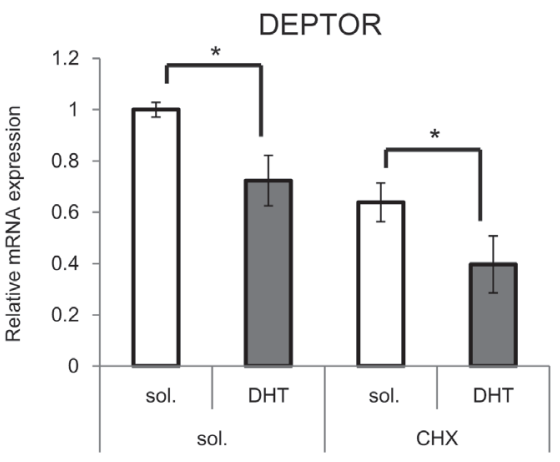

B

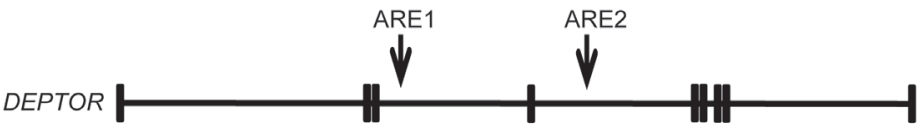

C

$$
\frac{\text { INPUT }}{\text { sol. } \quad \text { DHT }} \frac{\lg G}{\text { sol. } \quad \text { DHT }} \frac{\text { AR }}{\text { sol. DHT }}
$$

FKBP ARE

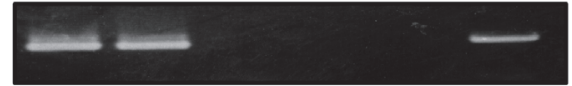

DEPTOR

Promoter

DEPTOR ARE1

DEPTOR ARE2
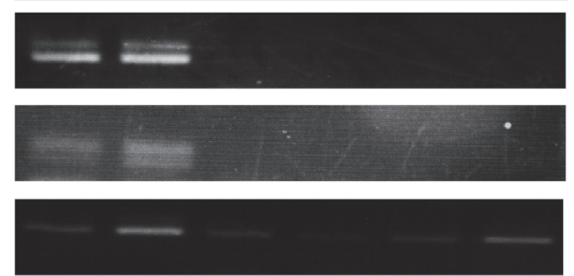

D

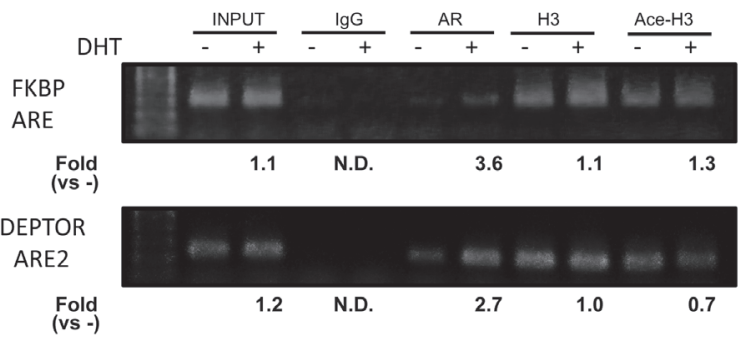

Fig. 3. Binding of AR to intronic ARE of DEPTOR gene. (A) LNCaP cells were treated with $10 \mu \mathrm{g} / \mathrm{mL}$ CHX. After $30 \mathrm{~min}$, cells were treated with solvent control (sol.) or $10 \mathrm{nM}$ DHT for $6 \mathrm{hr}$. Cells were harvested and the mRNA level of DEPTOR was measured by qRT-PCR. Results normalized against those of $\beta$-actin are expressed as averages of fold activation over the solvent control of four individual experiments (mean \pm S.D., $n=4) ; * P<0.05$. (B) Schematic representation of DEPTOR gene locus. Black boxes designate the exons. Two putative AR-binding regions (ARE1 and ARE2) were indicated by arrows. (C, D) LNCaP cells were treated with sol. or $10 \mathrm{nM}$ DHT for $24 \mathrm{hr}$. ChIP-experiment was performed using individual antibodies against $\mathrm{AR}$, histone $\mathrm{H} 3$ and acetylated histone $\mathrm{H} 3$ as well as control ones. The precipitated DNA was used for PCR amplification with the specific primers for ARE regions of DEPTOR gene and ARE region of FKBP5 gene used as a positive control. N.D., not detected.

regulates $E$-cadherin gene expression via recruitment of HDACs to ARE. Taken together, our observations suggest that downregulation of DEPTOR gene transcription was provoked by decreasing acetylated histone $\mathrm{H} 3$ levels.

In conclusions, we showed that DEPTOR gene expression was downregulated directly by AR and consequently activated mTOR/S6K-mediated cyclin D1 accumulation. The AR-DEPTOR-mTOR-S6K-cyclin D1 might be essential for the androgen-dependent cell proliferation in prostate cancer cells.

\section{ACKNOWLEDGMENTS}

This work was partly supported by MEXT KAKENHI [Grants 23790104 and 25460175].
Conflict of interest---- The authors declare that there is no conflict of interest.

\section{REFERENCES}

Bennett, N.C., Gardiner, R.A., Hooper, J.D., Johnson, D.W. and Gobe, G.C. (2010): Molecular cell biology of androgen receptor signalling. Int. J. Biochem. Cell. Biol., 42, 813-827.

Dehm, S.M. and Tindall, D.J. (2007): Androgen receptor structural and functional elements: role and regulation in prostate cancer. Mol. Endocrinol., 21, 2855-2863.

Duan, S., Skaar, J.R., Kuchay, S., Toschi, A., Kanarek, N., Ben-Neriah, Y. and Pagano, M. (2011): mTOR generates an auto-amplification loop by triggering the $\beta \operatorname{TrCP}-$ and $\mathrm{CK} 1 \alpha$ dependent degradation of DEPTOR. Mol. Cell., 44, 317-324.

Gao, D., Inuzuka, H., Tan, M.K., Fukushima, H., Locasale, J.W., Liu, P., Wan, L., Zhai, B., Chin, Y.R., Shaik, S., Lyssiotis, 
Y. Kanno et al.

C.A., Gygi, S.P., Toker, A., Cantley, L.C., Asara, J.M., Harper, J.W. and Wei, W. (2011): mTOR drives its own activation via $\mathrm{SCF}(\beta \operatorname{TrCP})$-dependent degradation of the mTOR inhibitor DEPTOR. Mol. Cell., 44, 290-303.

Guertin, D.A. and Sabatini, D.M. (2007): Defining the role of mTOR in cancer. Cancer Cell, 12, 9-22.

Heinlein, C.A. and Chang, C. (2004): Androgen receptor in prostate cancer. Endocr. Rev., 25, 276-308.

Laplante, M. and Sabatini, D.M. (2012): mTOR signaling in growth control and disease. Cell, 149, 274-293.

Lee, D.K. and Chang, C. (2003): Endocrine mechanisms of disease: Expression and degradation of androgen receptor: mechanism and clinical implication. J. Clin. Endocrinol. Metab., 88, 40434054.

Li, H., Sun, G.Y., Zhao, Y., Thomas, D., Greenson, J.K., Zalupski, M.M., Ben-Josef, E. and Sun, Y. (2014): DEPTOR has growth suppression activity against pancreatic cancer cells. Oncotarget, 5, 12811-12819.

Liu, Y.N., Liu, Y., Lee, H.J., Hsu, Y.H. and Chen, J.H. (2008): Activated androgen receptor downregulates E-cadherin gene expression and promotes tumor metastasis. Mol. Cell. Biol., 28, 70967108.

Matsumoto, T., Takeyama, K., Sato, T. and Kato, S. (2003): Androgen receptor functions from reverse genetic models. J. Steroid Biochem. Mol. Biol., 85, 95-99.

Matsumoto, T., Takeyama, K., Sato, T. and Kato, S. (2005): Study of androgen receptor functions by genetic models. J. Biochem., 138, 105-110.

Matsumoto, T., Shiina, H., Kawano, H., Sato, T. and Kato, S.
(2008): Androgen receptor functions in male and female physiology. J. Steroid Biochem. Mol. Biol., 109, 236-241.

Peterson, T.R., Laplante, M., Thoreen, C.C., Sancak, Y., Kang, S.A., Kuehl, W.M., Gray, N.S. and Sabatini, D.M. (2009): DEPTOR is an mTOR inhibitor frequently overexpressed in multiple myeloma cells and required for their survival. Cell, 137, 873-886.

van Duin, M., van Marion, R., Vissers, K., Watson, J.E., van Weerden, W.M., Schröder, F.H., Hop, W.C., van der Kwast, T.H., Collins, C. and van Dekken, H. (2005): High-resolution array comparative genomic hybridization of chromosome arm 8q: evaluation of genetic progression markers for prostate cancer. Genes Chromosomes Cancer, 44, 438-449.

Wang, Q., Li, W., Zhang, Y., Yuan, X., Xu, K., Yu, J., Chen, Z., Beroukhim, R., Wang, H., Lupien, M., Wu, T., Regan, M.M., Meyer, C.A., Carroll, J.S., Manrai, A.K., Jänne, O.A., Balk, S.P., Mehra, R., Han, B., Chinnaiyan, A.M., Rubin, M.A., True, L., Fiorentino, M., Fiore, C., Loda, M., Kantoff, P.W., Liu, X.S. and Brown, M. (2009): Androgen receptor regulates a distinct transcription program in androgen-independent prostate cancer. Cell, 138, 245-256.

Xu, Y., Chen, S.Y., Ross, K.N. and Balk, S.P. (2006): Androgens induce prostate cancer cell proliferation through mammalian target of rapamycin activation and post-transcriptional increases in cyclin D proteins. Cancer Res., 66, 7783-7792.

Zhao, Y., Xiong, X. and Sun, Y. (2011): DEPTOR, an mTOR inhibitor, is a physiological substrate of $\mathrm{SCF}(\beta \operatorname{TrCP}) \mathrm{E} 3$ ubiquitin ligase and regulates survival and autophagy. Mol. Cell, 44, 304316. 